\title{
Lidil
}

Revue de linguistique et de didactique des langues

46 | 2012

Typologie et description linguistiques

\section{Les classificateurs numéraux du stieng du Cambodge}

Noëllie Bon

\section{OpenEdition}

\section{Journals}

Édition électronique

URL : http://journals.openedition.org/lidil/3242

DOI : $10.4000 /$ lidil.3242

ISSN : $1960-6052$

\section{Éditeur}

UGA Éditions/Université Grenoble Alpes

\section{Édition imprimée}

Date de publication : 15 décembre 2012

Pagination : 45-77

ISBN : 978-2-84310-236-3

ISSN : $1146-6480$

\section{Référence électronique}

Noëllie Bon, «Les classificateurs numéraux du stieng du Cambodge », Lidil [En ligne], 46 | 2012, mis en ligne le 15 juin 2014, consulté le 01 mai 2019. URL : http://journals.openedition.org/lidil/3242 ; DOI

10.4000/lidil.3242

(c) Lidil 


\title{
Les classificateurs numéraux du stieng du Cambodge ${ }^{1}$
}

\author{
Noëllie Bon*
}

\begin{abstract}
RÉSUMÉ
Cet article traite du système de classificateurs en stieng (langue mônkhmer, groupe bahnarique sud), une langue peu décrite parlée au Vietnam et au Cambodge, dans une perspective typologique et aréale. La description s'attache dans un premier temps à souligner certains critères morphosyntaxiques, à savoir l'ordre des constituants, la distinction entre classificateurs et deux autres types de catégorisation nominale (noms de classe et classificateurs mensuraux), et les extensions fonctionnelles. Puis sont mentionnés différents aspects ayant trait à la catégorisation du système (inventaire, niveaux de catégorisation, origine), avec enfin une description sémantique détaillée.
\end{abstract}

\begin{abstract}
This paper considers the classifier system of Stieng (Môn-Khmer language, South-Bahnaric group), a very little-described language spoken in Vietnam and Cambodia, in a typological and areal perspective. The description first presents some morphosyntactic aspects of the system, namely the constituent order, the distinction between classifiers and two other types of nominal classification (class terms and mensural classifiers), and functional extensions. The study then examines some categorization aspects of the system (inventory, levels of categorization, origin), followed by a detailed semantic description.
\end{abstract}

1. Remerciements à Colette Grinevald, co-directrice de ma thèse au Laboratoire DDL (Université Lyon 2) pour les discussions autour de sa typologie; Scott DeLancey (University of Oregon), co-directeur de ma thèse pour ses suggestions ainsi que Deth Thach (Maitre de conférence à l'INALCO) pour sa synthèse sur les classificateurs du khmer. Toutes les éventuelles erreurs ou imprécisions sont néanmoins de ma propre responsabilité.

* Noëllie Bon est doctorante au Laboratoire DDL (UMR 5596, Université Lyon 2). 


\section{Introduction}

Ce papier décrit le système de classificateurs numéraux de la langue stieng ${ }^{2}$, une langue môn-khmère du groupe bahnarique-sud, dans une perspective typologique. Le stieng utilise en effet comme outil de catégorisation nominale des classificateurs numéraux, lorsqu'il s'agit de compter des objets. Cette description est effectuée dans un cadre fonctionnel typologique et souligne ainsi les processus de grammaticalisation impliqués dans l'existence de ces classificateurs.

Ci-dessous sont présentés quelques exemples du système dont il est question dans ce papier :

\begin{tabular}{|c|c|c|c|c|}
\hline $\begin{array}{l}h e j \\
\text { 1sG.S }\end{array}$ & $\begin{array}{l}\text { Pə:n } \\
\text { avoir }\end{array}$ & $\begin{array}{l}k \partial{ }^{\prime} n \\
\text { enfant }\end{array}$ & $\begin{array}{l}\text { famot } \\
\operatorname{dix}\end{array}$ & $\begin{array}{l}d u t \\
\text { CL.personne }\end{array}$ \\
\hline 'J'ai & enfa & - Eli.K & $\mathrm{MP} \# 10^{3}$ & \\
\hline
\end{tabular}

(2)

$\begin{array}{lllll}\text { hej } \quad \text { Po:n } & \text { gow } & \text { puan } & \text { bok } \\ \text { 1sG.S } & \text { avoir } & \text { vache } & \text { quatre } & \text { cL.tête } \\ \text { 'J'ai quatre vaches' }- \text { Eli.T-Vi-JN\#1 } & \end{array}$

$$
\begin{array}{lll}
\text { parej } & \text { famat } & \text { tiom } \\
\text { cigarette } & \text { dix } & \text { cL.tronc } \\
\text { 'dix cigarettes' - Eli.Kh-MP\#2 }
\end{array}
$$

Cette étude s'appuie sur des données collectées en 2010 et 2011 dans le village de Dey Kraham (région de Kratie, Cambodge) auprès de deux locutrices principales et quatre locuteurs occasionnels. Ces données proviennent non seulement de textes naturels courts ${ }^{4}(\mathrm{~T}-)$, mais aussi d'élicitations basées sur :

- des textes naturels (Eli.T-);

- des cours de khmer (élicitations collectées dans une démarche personnelle d'apprentissage de la langue stieng) (Eli.Kh-MP-);

2. Le stieng est une langue à tradition orale en danger, localisée au Cambodge et au Vietnam, dont le nombre exact de locuteurs est à ce jour méconnu. La communauté regroupe néanmoins environ 50000 membres sur les deux pays. Le stieng au Cambodge est sévèrement menacé, peu à peu remplacé par le khmer, langue officielle et nationale du pays.

3. La référence du corpus est structurée de la manière suivante: Type de donnée (élicitation, texte, stimuli...) (- Thème/Titre) (- initiales du locuteur) \# $\mathrm{n}^{\circ} \mathrm{de}$ phrase.

4. Les textes courts sélectionnés pour cet article sont au nombre de quatre et totalisent 10 minutes. 
- des stimuli visuels, à savoir :

- une compilation de 33 photographies d'objets du monde réel existants dans ce village, lesquels sont présentés par paires, triplets, etc. (Sti-CL-);

- des descriptions d'illustrations de livres pour enfants (8 textes) (Sti.Il-);

- des descriptions de 71 vidéos courtes (destinées à l'analyse de l'expression de la trajectoire) ${ }^{5}$ (Sti-TRAJ-);

- une liste de 300 mots élicités dans un contexte de comptage afin de vérifier la catégorisation en différentes classes sémantiques (Li-CL1; Li-CL2).

L'article expose dans un premier temps des aspects typologiques et aréaux des classificateurs, afin de situer le sous-type des classificateurs numéraux (section 2). Sont ensuite décrites des caractéristiques morphosyntaxiques du système (3), à savoir l'ordre des constituants (3.1), la nature des classificateurs (3.2) ainsi que différentes fonctions du système dans la langue (3.3). La partie suivante concerne des aspects de catégorisation du système (4) et présente ainsi l'inventaire des classificateurs du stieng (4.1), leur origine (4.2) et leurs niveaux respectifs de catégorisation (4.3). Une description sémantique est ensuite proposée (5), accompagnée de commentaires sur les variations idiolectales. Enfin, l'article conclut en donnant une ouverture sur des aspects aréaux et dynamiques de ce système (6).

Cette étude représente la première description détaillée du système de classificateurs de cette langue ${ }^{6}$ dans un cadre fonctionnel-typologique, abordant différents aspects du système. Elle se veut par ailleurs plus complète et plus structurée que les descriptions existantes pour les autres langues bahnariques, lesquelles n'entrent pas systématiquement dans un cadre typologique ${ }^{7}$.

5. Compilation de vidéos réalisées dans le cadre du projet Trajectoire - programme FR2559 de la Fédération de Typologie et d'Universaux Linguistiques (TUL) - Jean-Michel Fortis, Colette Grinevald, Alice Vittrant (resp.).

6. Le sketch grammatical du stieng proposé par Vera Miller ne dresse qu'une liste des classificateurs (1976:33-35).

7. Voir notamment David Thomas (1971 : 131-136) qui propose une liste des classificateurs du chrau et Sylvain Vogel (2006 : 31-54) qui s'inscrit dans une approche culioliste pour le phnong. 


\section{Aspects typologiques et aréaux}

\subsection{Types de catégorisation nominale et types de systèmes de classificateurs}

Parmi les diverses propositions de typologies des classificateurs ${ }^{8}$, cette étude se base sur la typologie de Colette Craig $(1992,1993)$ et Colette Grinevald $(1999,2000,2002,2004)^{9}$ qui considère les systèmes de classificateurs comme un type de catégorisation nominale parmi d'autres types, tels que les noms de classes (systèmes lexicaux - voir 3.2.1), les systèmes de classes nominales (comme ceux des langues bantoues) et les systèmes de genres, caractérisés par leurs systèmes d'accords grammaticaux. Les classificateurs se manifestent comme des morphèmes ou des mots additionnels qui n'apparaissent qu'une fois et qui ne font donc pas l'objet de phénomènes d'accord comme on peut le constater dans les systèmes de genres ou de classes nominales.

Au sein des systèmes de classificateurs de noms, on distingue par ailleurs 4 sous-types, selon des critères morphosyntaxiques, à savoir selon la nature de la tête auprès de laquelle les classificateurs se placent. On recense ainsi : les classificateurs nominaux, les classificateurs numéraux, les classificateurs verbaux et les classificateurs génitivaux, qui respectivement apparaissent attachés - ou dans des positions adjacentes - à des noms, à des numéraux, à des verbes ou à des possessifs ${ }^{10}$.

Parmi ces quatre types, le système du stieng est un système de classificateurs numéraux. Comme nous le verrons en 3.2, les classificateurs numéraux comprennent à leur tour deux sous types de classificateurs, des classificateurs de tri (ou vrais classificateurs) et des mensuraux (ou quantifieurs / mensuratifs $^{11}$ ) (Grinevald, 1999 : 111-112). Dans ce

8. Notamment, Joseph Greenberg (1972); Robert Dixon (1986) et Alexandra Aikhenvald (1998, 2000).

9. La même linguiste sous le nom de Craig jusqu'en 1996 puis de Grinevald. Voir aussi l'annexe par Colette Grinevald dans Goldwasser \& Grinevald, 2012).

10. Dans tous les cas, ce sont bien des noms qu'ils catégorisent. Notons qu'il existe par ailleurs des classificateurs de verbes qui catégorisent non pas des noms mais des verbes. On les trouve dans des langues d'Australie et d'Amérique (cf. Goldwasser \& Grinevald, 2012 : annexe).

11. Équivalents aux termes de mesure dans les langues dépourvues de classificateurs. 
papier, nous nous intéressons précisément aux classificateurs numéraux de tri du stieng ${ }^{12}$.

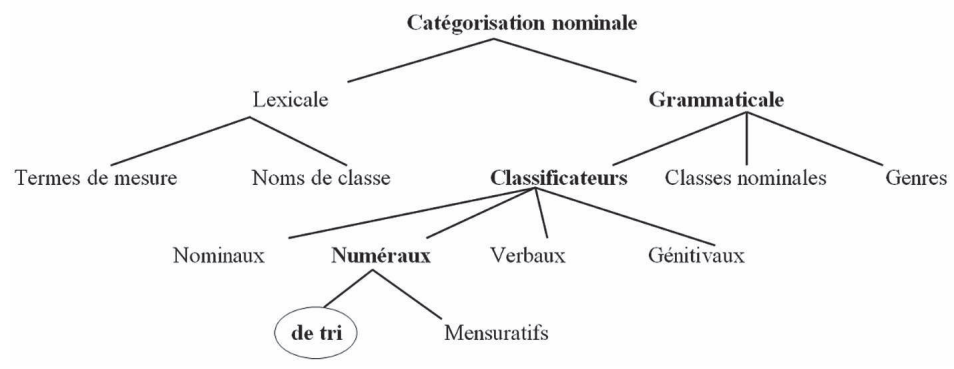

Figure 1. - Systèmes de catégorisation nominale ${ }^{13}$.

\subsection{Aspects aréaux des classificateurs numéraux}

L'utilisation de systèmes de classificateurs numéraux est un trait aréal des langues asiatiques. On en trouve également dans des langues d'Océanie et des Amériques ainsi que dans quelques langues d'Afrique. Ci-dessous, la carte du projet WALS (<http://wals.info $>$ ) de Leipzig illustre la distribution des classificateurs numéraux dans le monde, et témoigne de la densité de tels systèmes en Asie du Sud-Est (l'absence de classificateurs numéraux est notée par des marques blanches, leur présence optionnelle par des marques gris clair et leur présence obligatoire par des marques gris foncé).

Parmi les différents types de classificateurs, les classificateurs numéraux sont les plus courants et jusqu'ici les plus étudiés. En ce qui concerne ceux des langues d'Asie, on trouve en effet de nombreux travaux tels que ceux de Karen Adams \& Nancy Conklin (1973), Mary Erbaugh (1986), William Croft (1994) inter alia, ainsi que des sections au sein de descriptions, notamment sur des dialectes ou des langues apparentées au stieng comme le stieng-bulo (Miller, 1976), le bu-nong (phnong; Vogel, 2006), le chrau (Thomas, 1976) ou encore le khmer (Vogel, 2002; Haiman, 2010; Thach, c.p. 2012), une langue génétiquement plus éloignée mais qui par contact a considérablement influencé le stieng.

12. Pour des raisons de fluidité de lecture, nous les nommons par défaut les «classificateurs (numéraux) du stieng » dans le présent article.

13. Illustration schématique basée sur la typologie de Grinevald (2004) suite à des discussions avec l'auteur et avec son approbation. 


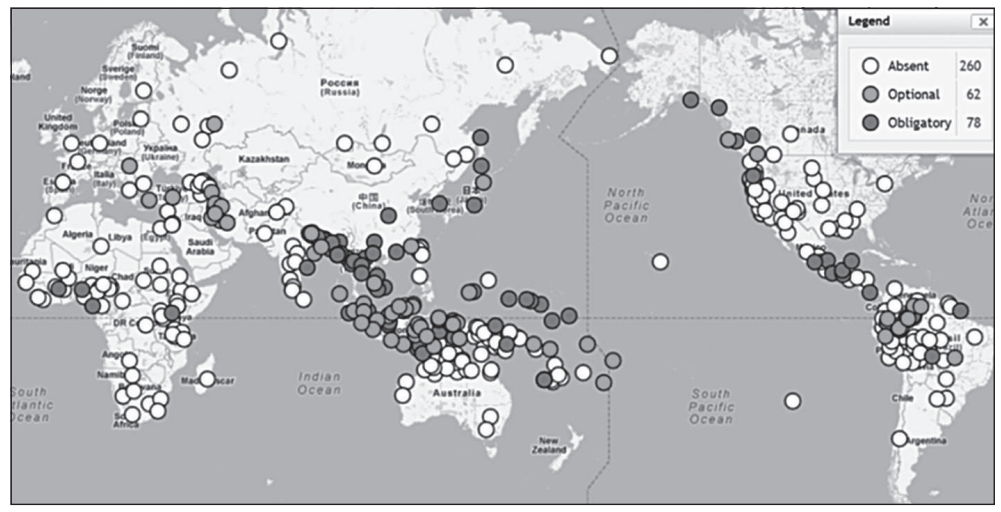

Carte 1. - Répartition aréale des classificateurs numéraux du WALS ${ }^{14}$.

Les écrits sur lesquels cette étude s'appuie principalement sont ceux de Walter Bisang $(1993,1999)$, qui traite notamment de la structure et de la fonction des classificateurs numéraux dans les langues d'Asie (voir 3.1 et 3.3), et Karen Adams (1982, 1986a et 1986b, 1989, 1992) qui propose une collection de travaux typologiques sur les classificateurs des langues austro-asiatiques en se basant principalement sur des critères sémantiques translinguistiques (cf. section 6).

\section{Classificateurs numéraux du stieng : morphosyntaxe et fonction}

\subsection{Ordre des constituants : classificateur postposé au numéral}

Les classificateurs du stieng, langue isolante, sont des morphèmes libres, l'ordre des constituants dans le groupe nominal étant $\mathrm{N}-\left[\mathrm{NUM}^{-}\right.$ $\mathrm{CL}]^{15}$, comme illustré en (4) :

14. Données cartographiques, (C) 2011 MapLink, Tele Atlas. D’après David Gil (2011).

15. D'après la typologie de Walter Bisang (1999: 118), il existe un modèle aréal asiatique des constructions à classificateurs selon un axe Nord-Sud : les langues du Nord asiatique ont tendance à adopter un ordre de type modifieurtête [NUM-CL]-N, tandis que les langues du Sud asiatique suivent l'ordre têtemodifieur N-[NUM-CL]. Le stieng, langue du Sud, est donc fidèle à ce modèle. 
(4)

\begin{tabular}{|c|c|c|c|c|}
\hline hej & Pa:n & $\begin{array}{l}\text { N } \\
k o r n\end{array}$ & $\begin{array}{l}\text { NUM } \\
\text { famət }\end{array}$ & $\begin{array}{l}\text { CL } \\
d u^{\prime}\end{array}$ \\
\hline$s G$ & avoir & enfant & dix & CL.personne \\
\hline
\end{tabular}

Notons que dans le groupe nominal, l'ordre prototypique est de type tête-modifieur, comme illustré en (5) :

$\begin{array}{llllll} & \mathbf{N} & \text { ADJ } & \text { ADV } & & \\ \text { Pən } & \boldsymbol{k}^{\boldsymbol{h}} \boldsymbol{\varepsilon j} & \boldsymbol{t e h} & \boldsymbol{m o t} & l 9 w & \text { trok } \\ \text { EXIST } & \text { lune } & \text { grand } & \text { très } & \text { sur } & \text { ciel }\end{array}$

'Il y a une très grande lune en haut dans le ciel' - Sti.Il-FRMM\#38

L'usage des classificateurs n'étant pas obligatoire, on note : N-[NUM (-CL)]. Leur absence ou leur présence serait vraisemblablement gouvernée par des critères pragmatiques encore non élucidés.

Sachant que les classificateurs ont une origine nominale (la plupart des noms en question faisant encore partie du lexique - cf. 4.2), le critère essentiel permettant de différencier un classificateur d'un nom est d'ordre syntaxique : le classificateur est toujours postposé au numéral tandis que le nom est toujours antéposé :

(6)
a. $\mathrm{N}$
NUM
CL
ko:n bair dui
enfant deux cl.personne

'Deux enfants'- Eli.Kh-MP\#5

b. $\mathrm{N}$ NUM CL

$\begin{array}{lll}\text { t9am\#pais } & \text { puan } & \text { t9am } \\ \text { cotonnier } & \text { quatre } & \text { cL.tronc }\end{array}$

'Quatre cotonniers'- Li-CL1\#177

$\begin{array}{lll}\text { c. } \mathrm{N} & \text { NUM } & \text { CL } \\ \text { parej } & \text { ba:r } & \text { t9əm } \\ \text { cigarette } & \text { deux } & \text { cL.tronc } \\ \text { 'Deux } & \text { cigarettes'- Eli.Kh-MP\#2 }\end{array}$

Une autre caractéristique syntaxique des classificateurs est leur «séparabilité ${ }^{16}$ » par rapport aux groupes nominaux : les constituants [NUM-CL] peuvent apparaitre en dehors du groupe nominal, séparés par un constituant extérieur au GN, comme un verbe ou une particule.

16. Cf. le cas du khmer où John Haiman parle de «separability» $(2010: 142,149)$. 
En (7), le constituant [NUM-CL] est séparé du nom par le verbe sow «voir». Dans cet exemple, ce constituant peut être considéré comme ayant une fonction d'anaphorique, reprenant le nom 'grenouille' (cf. 3.4.1). En (8), la tête nominale et le constituant [NUM-CL] sont séparés par une copule locative :

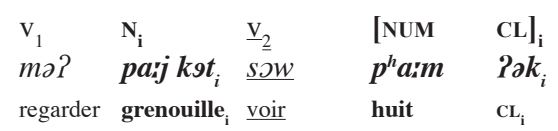

'En regardant les grenouilles, on en voit huit' / 'Des grenouilles, on en voit huit' - Sti.II-FS-MK\#76

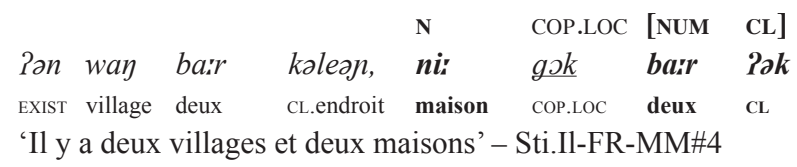

\subsection{Classificateurs vs autres types de classification nominale en stieng}

Les classificateurs du stieng sont à différencier de deux autres systèmes de catégorisation nominale : les noms de classe et les quantifieurs (ou classificateurs mensuraux).

\subsubsection{Classificateurs vs noms de classe}

\section{a) Classificateur et noms de classe : deux types de catégorisation nominale}

Comme dans la plupart des langues asiatiques, il existe un large inventaire de noms composés dans le lexique. On en recense plusieurs types parmi lesquels un type HYPERONYME+HYPONYME. L'hyperonyme dans ce cas-là représente un nom de classe dans la mesure où il catégorise le nom qui le suit en lui attribuant une classe particulière. Nonobligatoire, il peut néanmoins être requis pour lever des ambiguïtés, notamment dans les cas d'homophonie avec d'autres entrées lexicales ${ }^{17}$ - excepté lorsque le contexte du discours rend le sens du nom évident.

Voici dans le tableau 1 quelques exemples de noms composés avec des noms de classes appartenant au champ lexical des végétaux :

17. Comme dans le cas de pa:j\#be:h 'serpent' vs be:h 'vin' où le nom de classe paij 'animal' est requis pour différencier 'serpent' de 'vin'. 
(9) Arbres: toam \# N.spécifique ('tronc' \# N)

\begin{tabular}{|c|c|c|c|}
\hline a. & tэәm \# pais & tronc \# coton- & ‘cotonnier' Lex\#0480 \\
\hline 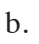 & tэəm \# pret & tronc \# banane & 'bananier' Lex\#0083 \\
\hline 、 & tэәт \# dü & tronc \# coco- & otier' \\
\hline
\end{tabular}

(10) Feuilles: lai \# N.spécifique ('feuille' \# N)

$\begin{array}{lllll}\text { a. } & \text { la: \# chi: } & \text { feuille \# bois } & \text { 'feuille' } & \text { Lex\#1525 } \\ \text { b. } & \text { la: \# pro.dit' } & \text { feuille \# épinard } & \text { 'épinard' } & \text { Lex\#1527 } \\ \text { c. } & \text { la: \# tuər } & \text { feuille \# oreille } & \text { 'oreille' } & \text { Lex\#0634 }\end{array}$

(11) Fruits: $\quad p \varepsilon j$ \# N.spécifique ('fruit'\# N)
a.
pej\# buət
fruit \# maïs
'maïs' Lex\#0198
b.
pej \# diap
fruit \# pomme
'pomme Lex\#0007
cannelle
cannelle'
c.
pej \# sa.wa:j
fruit \# mangue
'mangue' Lex\#1413

(12) Tubercules : mbum \# N.spécifique ('tubercule'\# N)

a. mbum \# kliam tubercule \# manioc 'manioc' Lex\#0057

b. mbum \# dom tubercule \# rouge 'radis' Lex\#0120

Tableau 1. - Noms de classe du stieng : champ lexical des végétaux.

Les classificateurs et les noms de classe appartiennent à deux systèmes de catégorisation nominale distincts : les noms de classe constituent un outil lexical de catégorisation (voir 2.1), par opposition aux classificateurs qui en constituent un outil grammatical.

Un premier argument est d'ordre morphosyntaxique : les noms de classes forment, avec leur composé, une unique tête nominale qui par ailleurs peut elle-même être modifiée par un numéral suivi d'un classificateur :

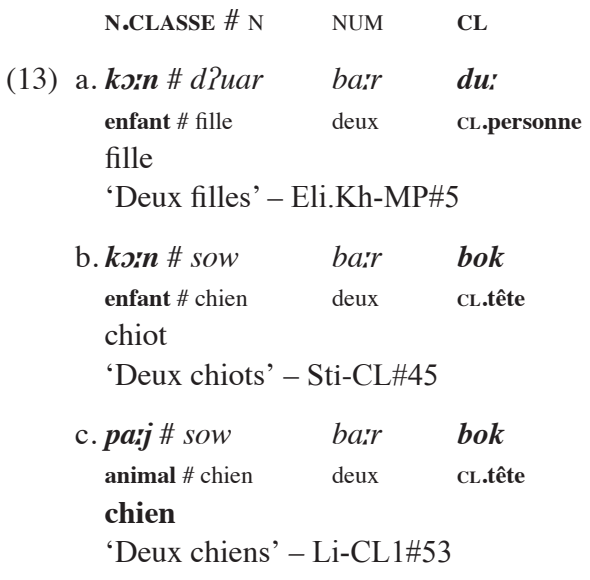




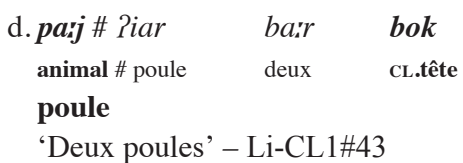

Certains exemples illustrent des cas d'homophonie entre nom de classe et classificateur comme en (14), ce qui justifie l'existence de deux systèmes distincts. Comme nous le verrons par la suite, ces exemples témoignent d'un phénomène d'écho entre nom de classe et classificateur :

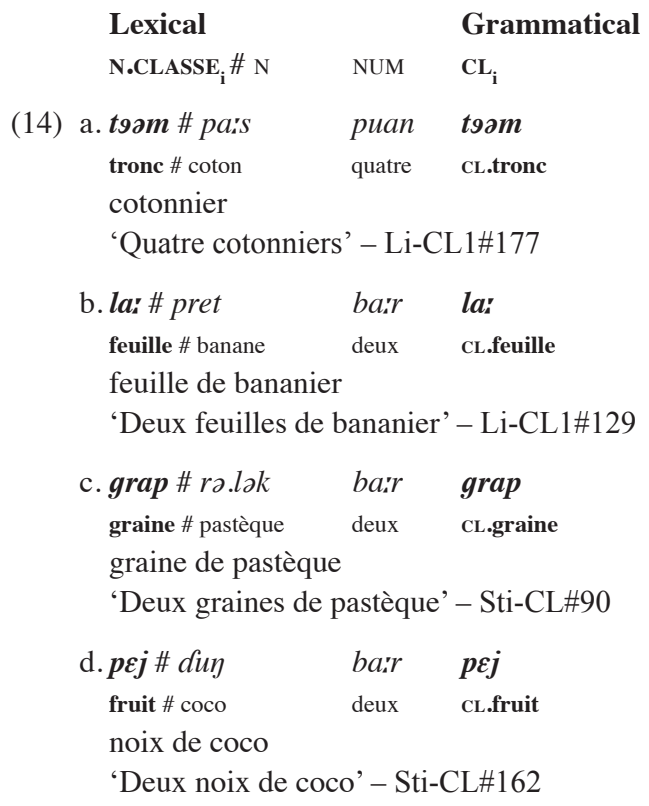

\section{b) Noms de classe et classificateurs dits «repeaters»}

Il existe un phénomène très répandu parmi les langues à classificateurs où les classificateurs ont une forme identique au nom qu'ils classifient dans la littérature, on parle de «repeaters» ou classificateurs échos pour labelliser ces classificateurs (Grinevald, 2004 : 1026). Ce phénomène est très rare dans les données du stieng : une seule occurrence, présentée en (15), est à ce jour présente dans le corpus :

$$
\begin{array}{lll}
\boldsymbol{c}^{h} \boldsymbol{e j} \quad \text { pej } & \boldsymbol{c}^{h} \boldsymbol{e j} \\
\text { corde trois } & \text { cL.corde } \\
\text { 'Trois cordes' } & - \text { Sti-CL\#37 }
\end{array}
$$


En revanche, comme nous l'avons évoqué avec les exemples présentés en (14), il existe des cas témoignant d'un phénomène d'écho entre classificateur et nom de classe. En d'autres termes, l'écho se produit non pas avec un nom simple ou une tête de nom composé (comme ce serait le cas pour un repeater prototypique) mais avec un morphème étant déjà lui-même un élément de classification, à savoir le nom de classe. Ces classificateurs particuliers semblent constituer une variante de repeater que nous nommons ici «class repeaters» ou classificateurs échos de classe. Ces exemples témoignent ainsi d'une sorte de phénomène en cascade où l'on aurait un premier système de classification situé directement sur le nom (nom de classe) et un second système postposé au numéral (classificateur).

Ce phénomène est assez courant en stieng, de même que dans des langues de la région telles que le birman (Vittrant, 2002 : 138) ou le thai (DeLancey, 1986 : 48). D'un point de vue diachronique et dynamique, Scott DeLancey (1986, 40 et 445-46) suggère que ce sont les noms de classe qui sont majoritairement à l'origine du développement des classificateurs numéraux : on a donc un premier système de classification, le système de noms de classe, qui semble se reproduire sur un système plus récent, le système de classificateurs numéraux.

Par ailleurs Karen Adams, citée par Colette Grinevald (2004 : 1026 [Traduction par Noëllie Bon]) constate «la récurrence très étendue d'un inventaire très restreint et spécifique de noms lexicaux qui a fait émerger des classificateurs partout dans le monde. Il s'agit de trois noms appartenant au domaine des plantes : arbre, feuille et fruit; le premier étant de loin la source la plus universelle de classificateur». Les données du stieng sont en accord avec ce constat :

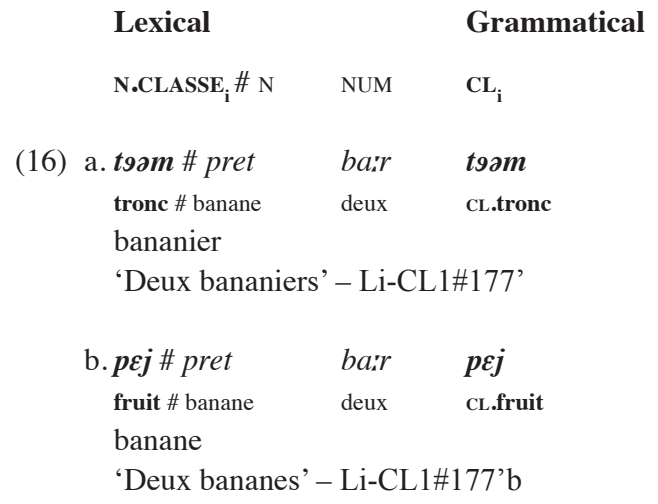




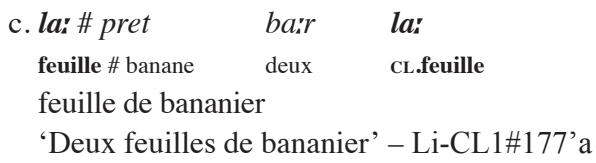

Comme nous le verrons par la suite, les classificateurs t9əm, la: et $p \varepsilon j$ représentent les classificateurs principaux des classes relatives à la forme des objets (5.2).

\subsubsection{Classificateur de tri vs classificateur mensural (quantifieur)}

Parmi les classificateurs numéraux, on distingue les classificateurs de tri des classificateurs mensuraux (ou quantifieurs). Plusieurs critères permettent de les différencier : des critères sémantiques (a) et des critères syntaxiques (b).

\section{a) Distinction sémantique ${ }^{18}$}

D'un point de vue sémantique, on distingue ces deux types de classificateurs sur la possibilité de quantifier une entité du monde réel soit en la comptant, soit en la mesurant. En ce sens, les classificateurs de tri sont utilisés pour individualiser des unités discrètes ${ }^{19}-$ le référent du nom tête ayant pour vocation d'être compté.

De plus, les classificateurs de tri catégorisent les référents dans des classes selon des caractéristiques qui leur sont inhérentes telles que les traits [ \pm animé], [ \pm humain], la forme, la fonction, etc. (cf. 5).

Les classificateurs mensuraux (quantificateurs), quant à eux, sont utilisés non seulement pour mesurer des unités non-discrètes (unités physiques ou non - i.e. liquides, matériaux, etc.), mais aussi des unités

18. Voir Colette Grinevald (2004: 1020 et 2002: 260-261) et résumé dans Alice Vittrant $(2002: 132)$ pour plus de précisions sur la différence sémantique entre classificateurs de tri et mensuraux.

19. Les noms référant à ces unités sont traditionnellement labellisés «noms comptables ou dénombrables» en grammaire du français et de count nouns en anglais. Par précaution, nous évitons d'employer ces termes, considérant que les langues ne délimitent pas forcément les noms comptables des noncomptables de la même façon: on part du principe qu'un référent donné pourrait très bien fonctionner comme un mass noun dans une langue $\mathrm{x}$ mais comme un count noun dans une langue $\mathrm{y}$. Tel est le cas en stieng pour les noms 'gingembre'; 'poivre'; 'sésame', etc. qui sont traités comme des count nouns, par opposition au français qui les traite comme des mass nouns. 
discrètes organisées en ensembles d'unités ${ }^{20}$. Comme le rappelle Colette Grinevald (2002 : 260-261), ces mensuratifs considérés comme des classificateurs mensuraux sont en fait présents dans toutes les langues du monde ${ }^{21}$.

Les classificateurs du stieng qui nous intéressent ici appartiennent à un système de classificateurs de tri. La différence sémantique entre les deux types de classificateurs de tri et mensuraux n'est pas toujours évidente. C'est pourquoi la syntaxe peut également être utile et pertinente pour les différencier.

\section{b) Distinction syntaxique}

D'un point de vue syntaxique, en stieng, les classificateurs de tri se comportent de la même façon que les mensuraux, dans la mesure où ils apparaissent exactement au même emplacement dans le groupe nominal; néanmoins, contrairement aux mensuraux dont l'usage est obligatoire, l'usage des classificateurs de tri est optionnel :

- dans le cas des noms référant à des unités non discrètes, l'omission du mensural qui les modifie rend la phrase agrammaticale, comme illustré en (17) :

\begin{tabular}{|c|c|c|c|c|}
\hline a. $h e j$ & Pən & berh & bair & dəə⿱亠䒑 \\
\hline *hej & ?әn & berh & bair & $\varnothing$ \\
\hline 1sG.S & & & 1) & \\
\hline
\end{tabular}

'J'ai deux bouteilles de vin' - Eli.T- Vi-JN\#5

\begin{tabular}{|c|c|c|c|}
\hline b. Paрәәs & $p^{h} e j$ & prih & grap \\
\hline *?apəəs & $p^{h} e j$ & porh & $\varnothing$ \\
\hline apporter & $\mathbf{r i z}^{22}$ & sept & \\
\hline
\end{tabular}

'J'ai apporté sept grains de riz' - Eli.T- Vi-JN\#6

- dans le cas des noms référant à des unités discrètes, l'omission du mensural modifie le sens de la phrase, comme illustré en (18) (on passe d'un processus de mesure à un processus de comptage); or,

20. Il s'agit de ce que l'on nomme traditionnellement en grammaire du français les noms indénombrables ou non comptables ou encore mass nouns en anglais. Pour les raisons explicitées plus haut, par précaution, nous n'utilisons pas ces termes dans la présente étude.

21. Comme verre/goutte/litre dans «un(e) verre/goutte/litre de vin» ou encore pile/rangée/carton dans «un(e) pile/rangée/carton de livres» en français.

22. Décortiqué et blanchi. 
ce n'est pas le cas lorsque l'on omet un classificateur dans une construction à classificateur (19) :

$\begin{array}{rll}\text { (18) a. porej } & \text { barr } & \text { kocap } \\ \text { *porcj } & \text { bair } & \varnothing \\ \text { cigarette } & \text { deux } & \text { ms.paquet }\end{array}$

'Deux paquets de cigarettes' - Eli.Kh-MP\#1

\begin{tabular}{|c|c|c|c|c|}
\hline b. $2 \partial n$ & tә:'m tәпиวt & bair & gəm & $d i \partial c$ \\
\hline *?วп & tə:m tәпиət & bair & & $d i \partial c$ \\
\hline EXIST & palmier & deux & Ms.bosquet & près \\
\hline
\end{tabular}

'Il y a deux bosquets de palmiers près de l'eau' - Eli.Sti.IlFR-MK\#8

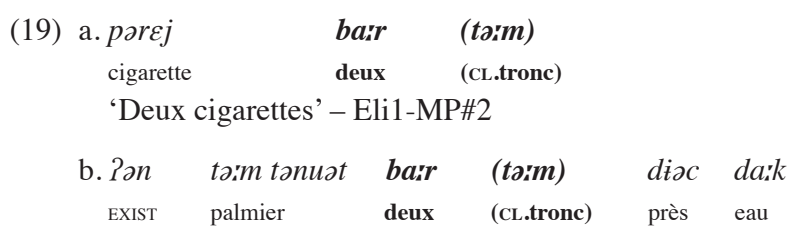

'Il y a deux palmiers près de l'eau' - Sti.Il-FR-MK\#8

\subsection{Fonction des classificateurs du stieng}

D'après Walter Bisang (1999 : 113), «la fonction des classificateurs (numéraux) est de rendre les noms comptables énumérables en les individualisant et en les classifiant, la fonction première de classification n'impliquant pas forcément le 'comptage'» [traduction par Noëllie Bon]. Comme nous l'avons déjà évoqué, l'opération de classification consiste à inclure une entité donnée dans une classe particulière en fonction des propriétés de cette entité.

Walter Bisang propose une typologie regroupant quatre types de langues selon les combinaisons de fonctions attestées dans lesdites langues (Bisang, 1999 : 117) :

$\begin{array}{ccc}\text { Classification } & \begin{array}{c}\text { Identification } \\ \downarrow\end{array} & \text { Individualisation } \\ & \text { Référentialisation } & \downarrow \\ & \text { Relationalisation } & \text { Comptage }\end{array}$

Figure 2. - Expansion fonctionnelle des classificateurs.

D'après Bisang (1999 : 115) [Traduction par Noëllie Bon]. 
Outre les fonctions de classification, d'identification et d'individualisation qui, d'après Walter Bisang, sont inhérentes à toutes les langues pourvues de classificateurs en Asie de l'Est et du Sud-Est (1999 : 116), les classificateurs du stieng sont vraisemblablement caractérisés par des fonctions de référentialisation et de relationalisation. Ils semblent également porter une fonction discursive - qui expliquerait notamment le caractère facultatif des classificateurs en stieng - mais celle-ci n'est pas exposée dans le présent papier car encore non élucidée.

\subsubsection{Fonction de référentialisation : usage anaphorique}

D'après William Croft (1994 : 163), une tendance aréale des langues à classificateurs en Asie est l'usage anaphorique de ces classificateurs (reference tracking), phénomène que l'on retrouve en stieng :
a. $m \partial ?$
paij kst
sow
$p^{h}$ aim
$\boldsymbol{P a k}_{i}$ regarder grenouille voir huit $\mathrm{CL}_{\mathrm{i}}$

'En regardant les grenouilles, on en voit huit' - Eli.Il-FS-MK\#76
b. Pon sorh salar ${ }_{i}$ han rian pej $\boldsymbol{d} \boldsymbol{u}_{i}^{\prime}$, EXIST écolier ${ }_{i}$ aller apprendre trois cL.personne ${ }_{i}$ $\begin{array}{lllll}\boldsymbol{m}=\boldsymbol{b} \boldsymbol{u}_{j}^{\prime} & P a:=m a t & \boldsymbol{b a r r} & \boldsymbol{d} \boldsymbol{u}_{y}^{\prime} & P a:=k i \partial j \\ \text { un=cL.personne, } & \text { côté=devant } & \text { deux } & \text { cL.personne } & \text { côté=derrière }\end{array}$ 'Il y a trois écoliers ${ }_{i}$ qui partent apprendre, $\mathbf{u n}_{\mathbf{j}}$ devant, deux $_{\mathrm{y}}$ derrière' $^{23}$ - Eli.Il-FR-MM\#18

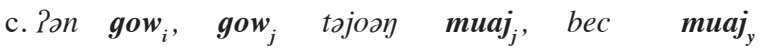 EXIST $\mathbf{v a c h e}_{\mathbf{i}}$ vache $_{\mathbf{j}}$ ê.debout un $\mathbf{u n}_{\mathbf{j}}$ ê.allongé un $\mathbf{u}_{\mathbf{y}}$ '[...] il y a des vaches ${ }_{i}$ : une vache ${ }_{j}$ debout, une couchée' - Eli.Il-FR-MM\#46

$\mathrm{Au}$ vu des données collectées à ce jour, il semble que cet usage soit réservé en stieng aux entités [+humaines]. En effet, dans le cas des entités [-humaines], le classificateur étant facultatif comme en (20c), on ne peut pas attribuer cette fonction au simple classificateur, mais au groupe numéral complet. Tandis que dans le cas des humains (20b), il semblerait que le classificateur soit obligatoire ${ }^{24}$.

23. $A v e c j+y=i$.

24. Davantage d'élicitations avec d'autres locuteurs doivent être réalisées pour le justifier. 


\subsubsection{Fonction de relationnalisation}

Les classificateurs utilisés pour les humains semblent dans certains cas avoir un usage de comitatif, avec le numéral 'deux' :

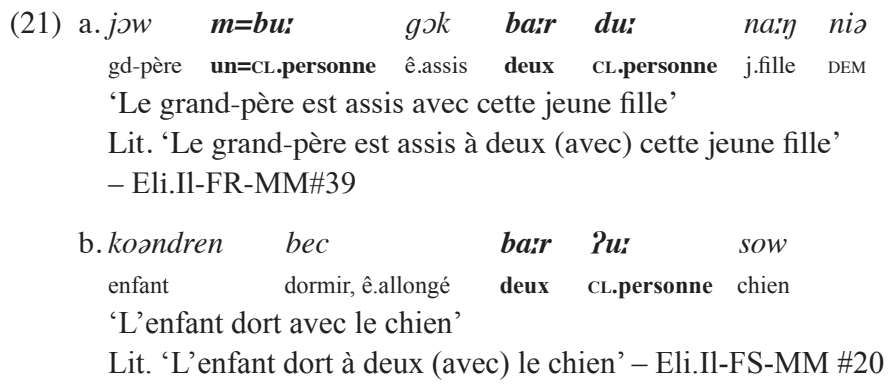

Remarque : en (21), le groupe numéral 'deux-CL.personne' peut s'exprimer par deux formes différentes, dont une semble être une réduction phonétique : bair du' et barr ?ui. Cette réduction n'est opérée qu'après le numéral ba:r 'deux'.

\section{Principes de catégorisation des classificateurs du stieng}

Cette section décrit les principes de catégorisation des classificateurs du stieng en présentant dans un premier temps leur inventaire, puis leur origine lexicale et enfin leur organisation sur différents niveaux de catégorisation.

\subsection{Inventaire des classificateurs du stieng}

Le stieng compte 15 classificateurs dans son système ${ }^{25}$. Ces derniers sont listés dans le tableau 2 et ordonnés par trait sémantique.

Il est par ailleurs important de donner quelques précisions sur les numéraux et leur co-occurrence avec les classificateurs :

- tous les classificateurs semblent pouvoir être utilisés avec tous les chiffres ${ }^{26}$, excepté le numéral $d i$ - 'un' qui ne peut pas apparaitre avec $? \partial k^{27}$;

25. Avec des variations idiolectales qui ne sont pas précisées ici.

26. Nous ne savons néanmoins pas encore si les classificateurs peuvent être utilisés avec des nombres au-delà de la centaine ou du millier.

27. Ce qui est cohérent d'un point de vue sémantique, ?ək marquant, dans son emploi lexical, la pluralité. 


\begin{tabular}{|c|c|c|c|c|c|c|c|c|c|c|c|c|c|c|}
\hline$\frac{n}{0}$ & 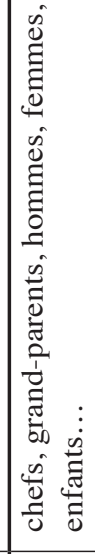 & 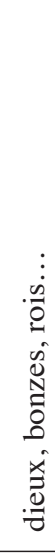 & 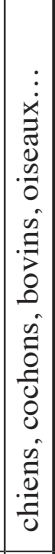 & 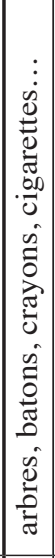 & 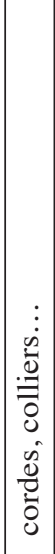 & 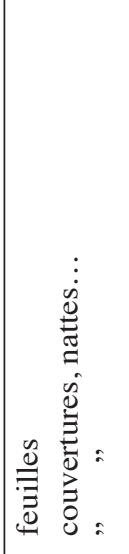 & $\stackrel{\mathscr{\Xi}}{\Xi}$ & 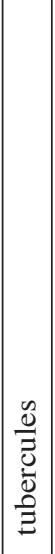 & 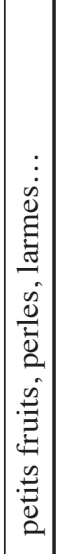 & 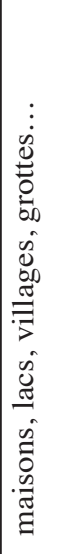 & 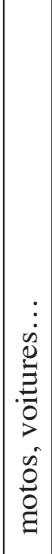 & 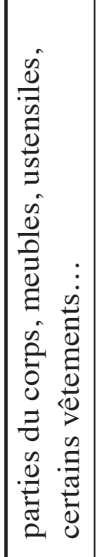 & 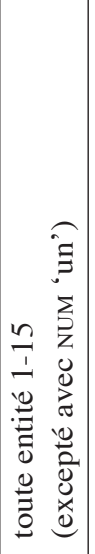 & வ் \\
\hline 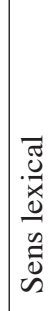 & 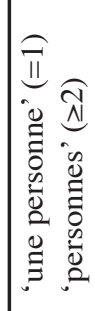 & iv & ¿ & 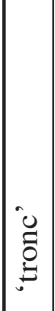 & 吾 & 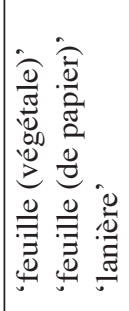 & : & 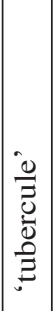 & : & . & 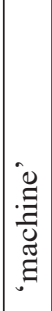 & & 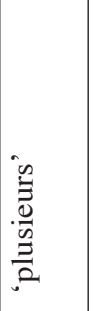 & 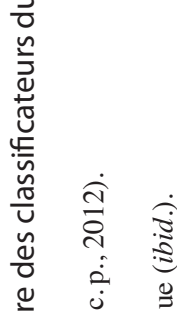 \\
\hline 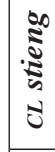 & : & $\underset{*}{\mathbb{S}}$ & $\Xi$ & 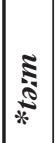 & : & 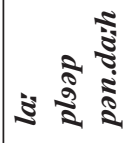 & $\Phi$ & $\begin{array}{c}\vdots \\
\vdots \\
\vdots \\
\vdots\end{array}$ & $\mid$ & 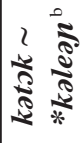 & 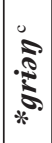 & $Q$ & $\lesssim$ & 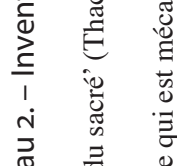 \\
\hline z & -2 & $m$ & $\nabla$ & in & 6 & $\wedge \infty a$ & 요 & $\approx$ & $\approx$ & 2 & \pm & 1 & 7 & 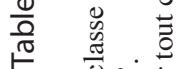 \\
\hline $\begin{array}{l}0 \\
0 \\
0 \\
\frac{\Xi}{U}\end{array}$ & 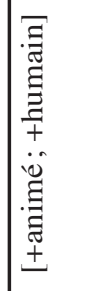 & & 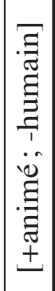 & 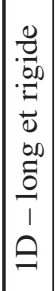 & 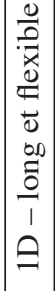 & 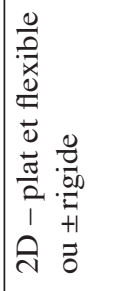 & 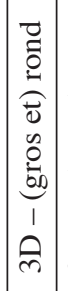 & 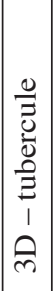 & 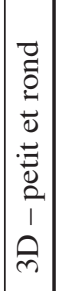 & 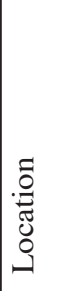 & 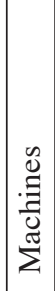 & 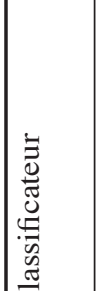 & 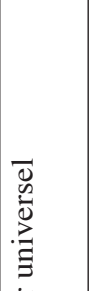 & 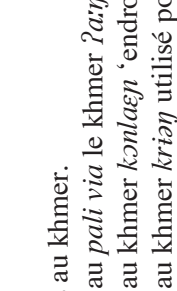 \\
\hline 霆 & 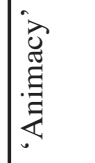 & & & 当 & & & & & & 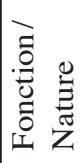 & & $\mid \begin{array}{l}0 \\
0 \\
\ddot{u} \\
\tilde{\Xi} \\
0 \\
\dot{\alpha}\end{array}$ & 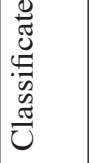 & 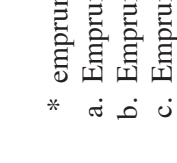 \\
\hline
\end{tabular}


- comme déjà précisé, il existe deux formes pour le numéral 'un' selon que l'on utilise ou non un classificateur: on a $d i$-CL et mиај+Ø. On utilise $d i$ - avec toutes les classes sauf la classe des [+humains] où $m b u^{28}$ est employé;

- la forme ba:r ?ak (NUM 'deux' + CL.universel) est souvent contractée en brok:

Le tableau 3 illustre les différentes formes de numéraux commentées ci-dessus et leur co-occurrence avec les classificateurs :

\begin{tabular}{|c|c|c|c|c|c|}
\hline Glose & $\mathrm{N}$ & NUM CL & Glose & $\mathrm{N}$ & NUM CL \\
\hline 'un buffle' & krəbit: & $\begin{array}{l}\text { тиај Ø } \\
* d i=? \partial k \\
\text { di=bok } \\
\text { *mиаj bok }\end{array}$ & 'deux buffles' & $k r \partial b i$ & $\begin{array}{l}\text { ba:r } \varnothing \\
\text { ba:r } ? a k \sim \text { brək } \\
\text { ba:r bok }\end{array}$ \\
\hline 'une personne' & sadiay & $\begin{array}{l}\text { mиај Ø } \\
* d i=? ə k \\
m=b u i \\
* \text { muaj bu: } \\
* d i=d u:\end{array}$ & 'deux personnes' & sadiay & $\begin{array}{l}\text { ba:r Ø } \\
\text { ba:r } 1 \partial k \sim \text { brək } \\
\text { ba:r dui' } \\
\text { Eli.Kh-MP\#0 }\end{array}$ \\
\hline
\end{tabular}

Tableau 3. - Formes des numéraux et co-occurrence avec les classificateurs.

\subsection{Origine nominale des classificateurs du stieng}

L'origine des classificateurs du stieng est aisément reconnaissable : ces derniers proviennent de noms qui sont encore utilisés dans le lexique. Il existe néanmoins une exception : le classificateur universel $\supsetneq ə k$ qui, dans son emploi lexical, est un adverbe ou un adjectif marquant la pluralité.

Le tableau 4 illustre quelques formes dans leur emploi lexical.

Ainsi, en stieng, le même morphème peut être utilisé dans différentes constructions : simple nom (ou nom composé), nom de classe et classificateur. Les langues d'Asie du Sud-Est étant isolantes, «les formes lexicales ne peuvent être identifiées comme appartenant à une classe fonctionnelle particulière mais peuvent apparaitre dans plusieurs

28. mbu' est vraisemblablement une contraction de mиаj bu', 'un CL.personne'. 


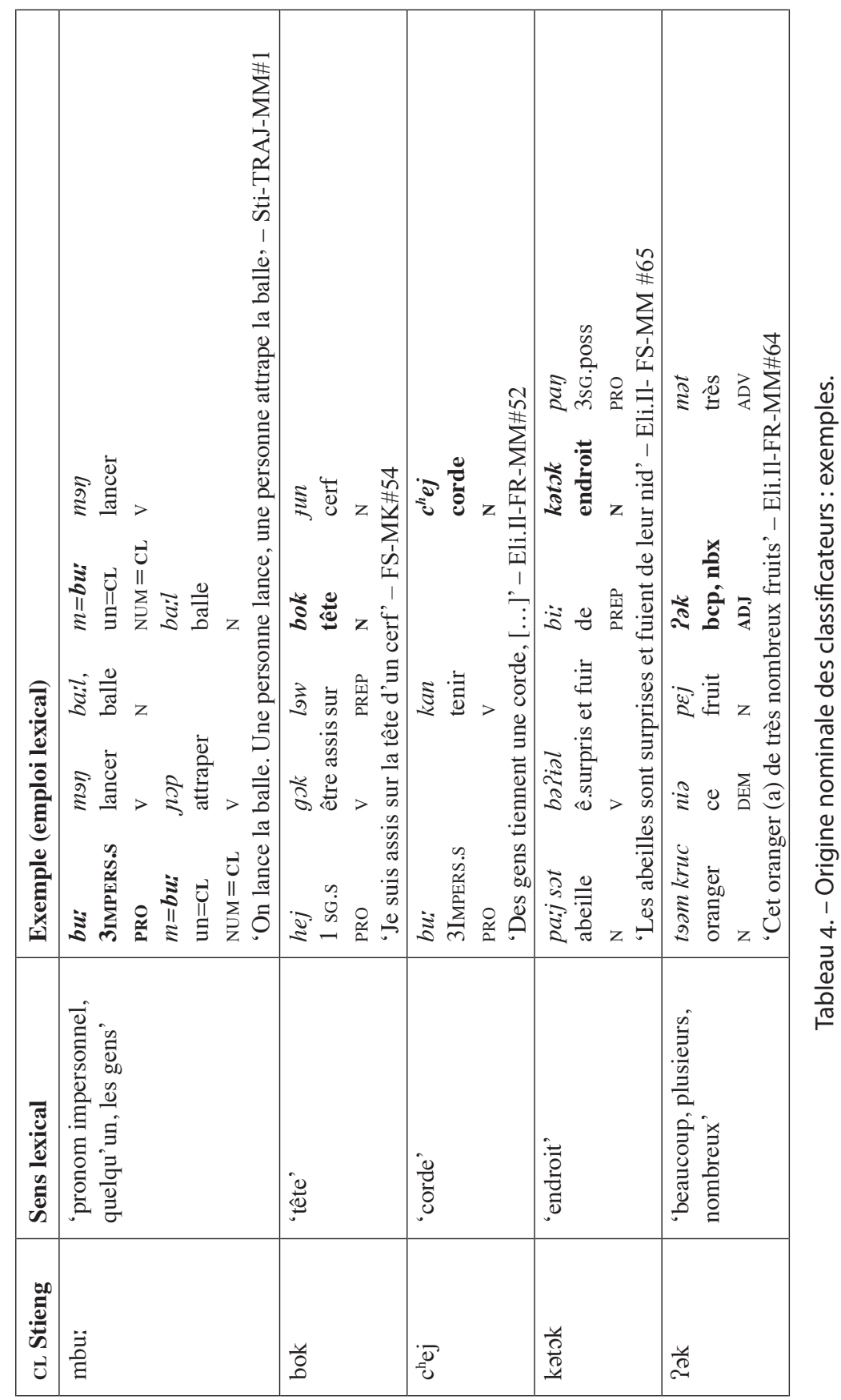


fonctions grammaticales » (Adams, 1986b : 244-245) [traduction par Noëllie Bon]. Pour les différencier des autres, comme souligné plus haut (3.1), on repère les classificateurs par leur position postposée au numéral.

\subsection{Niveaux de catégorisation ${ }^{29}$}

On reconnait différents niveaux de catégorisation des classificateurs, selon la taille de la classe qu'ils catégorisent et la relation sémantique qu'ils entretiennent avec les noms qu'ils classifient. On distingue ainsi les classificateurs suivants, selon leur portée de classification :

unique (4.3.3) << spécifique (4.3.2) << général (4.3.1) >> par défaut (4.3.4) (voir l'annexe de Goldwasser \& Grinevald, 2012).

\subsubsection{Classificateurs généraux}

Les classificateurs généraux catégorisent les noms au niveau taxonomique et peuvent classifier tous les membres du taxon en question. Dans le système du stieng, le classificateur pour les objets $3 \mathrm{D}, p \varepsilon j$ (cL.fruit - cf. section 5.2.3 sous-section a.) peut être utilisé avec tous les noms se référant à des fruits. De même, $m b u:(=1)$ et $d u:(\geq 2)$ classifient tous les humains (cf. 5.1.1); le classificateur bok (cL.tête - cf. 5.1.2) catégorise tous les noms d'animaux ${ }^{30}$, etc.

\subsubsection{Classificateurs spécifiques}

Les classificateurs spécifiques catégorisent des classes plus restreintes : en stieng, parmi les objets $3 \mathrm{D}$, il existe une classe spécifique pour les tubercules catégorisée par le classificateur mbum (CL.tuber - cf. section 5.2.3, sous-section b).

\subsubsection{Classificateurs uniques}

Les systèmes à classificateurs attestent souvent des classificateurs dits uniques qui catégorisent des classes avec un seul membre, tel que chyak en thaï qui ne classifie que le nom pour 'éléphant', dans un contexte honorifique (Grinevald, 2004 : 1017). À ce jour, les données du stieng n'attestent aucun cas de classificateur(s) unique(s).

29. Cette section s'appuie sur la partie 2.1 (Levels of categorization) de l'annexe proposée par Grinevald (Basics on Classifier Systems), dans Goldwasser \& Grinevald (2012).

30. Excepté les animaux considérés comme dépourvus de tête, voir 5.1.2. 


\subsubsection{Classificateur universel (par défaut)}

Enfin, dans de nombreux systèmes de classificateurs numéraux, on trouve un classificateur par défaut qui ne porte pas de valeur sémantique 'catégorisante'. Ce classificateur peut remplacer tous les autres ${ }^{31}$. En stieng, il s'agit du classificateur $2 \jmath k$, qui dans son emploi lexical est un adjectif marquant la pluralité.

Notons par ailleurs que de nombreux objets n'ont pas de classificateur propre, tels que les noms référant aux parties du corps (mains, doigts, pieds, etc.), aux ustensiles de cuisine (cuillère, couteau, gamelles, verres, mortier, etc.), aux vêtements et hamacs, aux animaux considérés comme 'sans tête', etc. - comme illustré dans les exemples (22) et (23). Les locuteurs ont alors deux possibilités : soit ils utilisent le classificateur universel $2 \jmath k$ lorsque le numéral est supérieur à un, soit ils n'en utilisent pas du tout.

(22)
a. $m$ ra:m\#ti:
pram (Pak)
doigt\#main
cinq
(CL)
'Cinq doigts' - Sti-CL\#1
b. sbeək\#fə刀
puan
$(9 ə k)$
chaussure
quatre
(CL)
'Quatre chaussures' - Sti-CL\#18

$\begin{array}{lllll}\text { c. } k a: & \text { bair }(\mathbf{P a k}) & t \varepsilon ? & P u: & \text { fa:m } \\ \text { poisson } & \text { deux (cL) } & \text { poser } & \text { PREP } & \text { assiette } \\ \text { 'Deux poissons (cuits) } & \text { posés dans l'assiette' } & \text { - Sti-CL\#3 }\end{array}$
(23) a. $k^{\text {ha:p }}$ da:k muaj $\varnothing$
jarre eau un $\varnothing$
'Une jarre à eau' - Sti-CL\#6

b. ncian mиaj $\varnothing$

bague un $\varnothing$

'Une bague' - Sti-CL\#1bis

\section{Classes sémantiques des classificateurs du stieng}

Globalement, d'un point de vue sémantique, le système est fidèle à la typologie de Adams \& Conklin (1973) : il s'organise selon deux cri-

31. Il correspondrait à utiliser les termes 'machin' ou 'truc' en français : par exemple, littéralement, (22a) serait traduit «doigts cinq trucs». 
tères principaux qui reposent sur le trait [ \pm animé] (critère $1-5.1$ ) et des paramètres physiques, principalement la forme et par extension la dimensionnalité (critère $2-5.2$ ). Le système est aussi caractérisé par des classificateurs relatifs à la fonction ou la nature des objets (5.3).

Il existe quelques variations idiolectales concernant l'usage de tel ou tel classificateur ou encore l'appartenance d'une entité à telle ou telle classe. Ces variations sont précisées au fil de la description sémantique.

\subsection{Critère $1:$ [+animé]}

\subsection{1. [+animés, thumains] : mbu:, du: - 'cl.personne'}

Il existe deux classificateurs différents selon le nombre d'individus, différenciant une entité de plusieurs entités: $m b u$ ( un); $d u:^{32}(\geq 2)$.

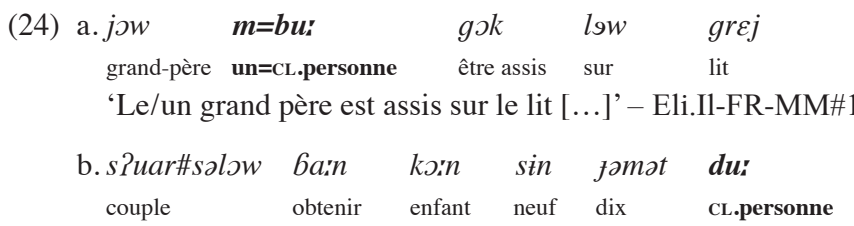

'Notre couple a eu neuf-dix enfants' - T-Vi-JN\#5

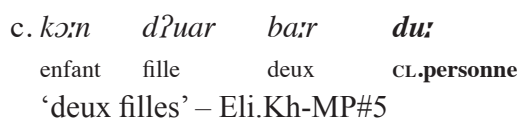

Ce système ne catégorise pas la classe socioculturelle et religieuse des entités comptées, contrairement aux systèmes de nombreuses langues de la région (Adams, 1992) - voir exemples (25a-f). Par ailleurs, les entités qui n'appartiennent pas au monde réel (fantômes, génies, dieux, morts, etc.) peuvent être classifiées avec ces mêmes classificateurs (ibid.) - voir exemples (26a-c). Il existe néanmoins un classificateur $P a: \eta^{33}$ 'CL.sacré', qui peut être utilisé avec les noms pour 'roi' (25a) et 'dieu' (26a) :

32. Remarque : comme déjà évoqué, le classificateur $d u$ : peut-être réalisé $? u$ : (réduction phonétique). La forme $d u$ : est par ailleurs utilisée comme pronom impersonnel et nom de classe (voir 4.2).

33. Rappel : emprunt au khmer Pa: $\eta$, classe du sacré. L'utilisation de ce classificateur est caractérisée par une variation inter-locuteurs: certains l'utilisent systématiquement tandis que d'autres ne l'utilisent pas du tout. Ceci témoigne vraisemblablement du degré de «khmérisation» du locuteur, par- 
(25)
$\begin{array}{cll}\text { a. } s d a c & \boldsymbol{m}=\boldsymbol{b u} \boldsymbol{i}^{\prime} & \left(\sim \text { di.Pa: }^{\prime} \eta\right) \\ \text { roi } & \text { un=cL.personne } & (\sim \text { un=CL.dieu })\end{array}$
'Un roi' - Li-CL2\#4
b. [...] lok gru: $\quad \boldsymbol{m}=\boldsymbol{b u}$ :
[...] professeur un=cL.personne
'[...] Un professeur' - Eli.Il-FR-MM\#30
c. nak\#tain $\quad \boldsymbol{m}=\boldsymbol{b u}$ '
tisserand un=CL.personne
'Un tisserand' - Li-CL11\#80
d. $\min \quad \boldsymbol{m}=\boldsymbol{b u} \boldsymbol{u}^{\prime}$
tante un=cL.personne
'Une tante' - Li-CL11\#83
e. kəan\#dren $\boldsymbol{m}=\boldsymbol{b u}$ :
bébé un=cL.personne
'Un bébé' - Li-CL11\#82
f. nak\#du's $\quad \boldsymbol{m}=\boldsymbol{b u} \boldsymbol{u}^{\prime}$
prisonnier un=cL.personne
'Un prisonnier' - Li-CL11\#81
(26) a.braih m=bur ( di.?a:' )
dieu un=cl.personne $(\sim$ un=CL.sacré $)$
'Un dieu' - CL-MM\#1
b. sadiay $c^{h} \partial t \quad \boldsymbol{m}=\boldsymbol{b u} \boldsymbol{i}$
stieng mort un=cL.personne
'Un mort' - CL-MM\#3
c. nak\#tar $\quad \boldsymbol{m}=\boldsymbol{b u}^{\prime}$
génie un=cL.personne
'Un génie' - CL-MM\#2

\subsection{2. [tanimés; -humains] : bok - 'cl.tête'}

On utilise en règle générale le classificateur bok, dont l'origine nominale signifie 'tête', avec les animaux, comme illustré en (27a-b) :

ticulièrement lorsque - comme dans ce cas précis - le nom fait référence à une entité culturellement très marquée. En effet, les Stieng sont traditionnellement animistes, mais aujourd'hui, la plupart ont été convertis au bouddhisme par les Khmers et pratiquent désormais un syncrétisme religieux, d'où l'emprunt du classificateur pour 'dieu' au khmer. 
(27)

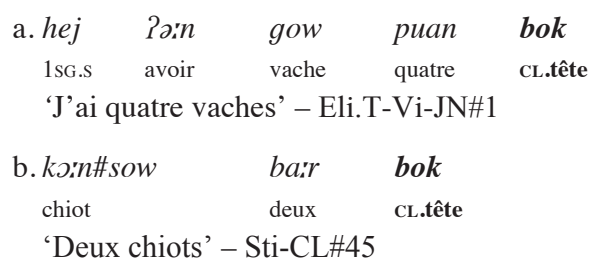

Néanmoins, il existe des nuances et l'on ne peut l'employer avec tous les animaux :

- l'utilisation ou non du classificateur bok semble être liée à la perception que les locuteurs ont d'un animal comme ayant une tête ou non : pour certains, les poissons, les fourmis, les sangsues, etc., n'auraient pas de tête, mais les araignées et les abeilles, les oiseaux et mammifères en auraient une ${ }^{34}$;

- une locutrice plus âgée utilise bok 'Cl.tête' pour compter les enfants mais d'autres locuteurs estiment que c'est impoli et ne l'utilisent que pour les animaux. Il s'agit là d'un phénomène de reclassification;

- dans les contes, les animaux sont souvent personnifiés et l'on utilise comme classificateur non pas bok, mais celui ordinairement utilisé pour les entités [+humaines] :

$\begin{array}{lllll}\begin{array}{l}\text { koəndren } \quad \text { bec } \\ \text { enfant }\end{array} \quad \begin{array}{l}\text { dormir, ê.allongé } \\ \text { 'L'enfant dort avec le chien' }\end{array} & \begin{array}{l}\text { deux } \\ \text { cL.personne }\end{array} & \begin{array}{c}\text { sow } \\ \text { chien }\end{array} \\ \text { Lit. 'L'enfant dort à deux (avec) le chien' - Eli.Il-FS-MM\#20 }\end{array}$

\subsection{Critère 2 :forme (paramètre physique pour les [-animés])}

Le second critère sémantique principal pour inventorier les classificateurs correspond à la forme, c'est-à-dire l'extension des objets dans les trois dimensions spatiales (1D-long, 2D-plat, 3D-rond). Ce critère est accompagné de paramètres secondaires, tels que la rigidité, la flexibilité ou la taille relative. Au sein de ce second critère, le système est surtout basé sur une taxonomie biologique, utilisant notamment des noms rela-

34. Lors des tests de grammaticalité avec des structures telles que *ka: barr bok 'trois poissons', une des locutrices corrige en ka: ba:r Ø, en justifiant qu'un poisson n'a pas de tête : le sens du nominal à l'origine du classificateur est encore actif. 
tifs aux végétaux (ou à des parties de végétaux) comme classificateurs. Une des explications à cet emploi métaphorique est que les végétaux sont traditionnellement utilisés comme matériau de base pour la plupart des objets du quotidien des locuteurs de ces langues (Erbaugh, 1986 : 430).

\subsubsection{Objets $1 D$ - longs}

a) Longs et rigides : tgam - 'cL.tronc'

On utilise le classificateur t9əm - dont l'origine nominale signifie 'tronc' - pour les objets longs et rigides.

(29) a.t9am\#pars puan t9әm

cotonnier quatre cL.tronc

'Quatre cotonniers' - Li-CL11\#177

b. klim bair t9am

piquet deux cL.tronc

'Deux piquets' - Sti-CL\#32

c. bec puan t9am

crayon quatre cu.tronc

'Quatre crayons' - Sti-CL\#36

d. parcj pej toam

cigarette trois cl.tronc

‘Trois cigarettes' - Sti-CL\#35

b) Longs et flexibles : $c^{h} e j-' c L . c o r d e^{\prime}$

On utilise le classificateur $c^{h} e j$, dont l'origine nominale signifie 'corde', pour les objets longs et flexibles : lianes, colliers, etc.

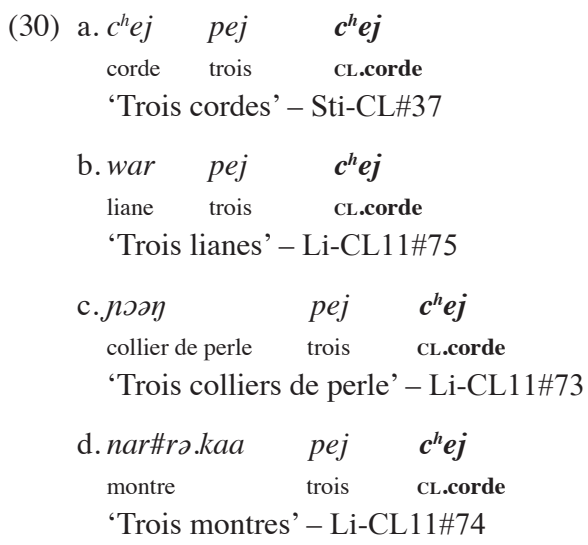




\subsubsection{Objets $2 D$ - plats et flexibles}

plsəp - 'CL.feuille' (papier) > panda:h - 'CL.lanière' > la: - 'CL.feuille' (végétale)

Le stieng utilise un classificateur provenant du nom la: 'feuille végétale' pour classifier les objets $2 \mathrm{D}$ plats et flexibles. la: est néanmoins exclusivement utilisé avec des noms renvoyant à des végétaux. On emploie plsap ('feuille de papier') et panda:h ('lanière') 35 pour des entités d'origine végétale mais modifiées par l'homme (tissus, nattes, etc.). On ne peut pas employer ces classificateurs avec tous les objets plats et flexibles, notamment des hauts et pantalons. D'autres nuances qui ne sont pas détaillées ici existent par ailleurs.

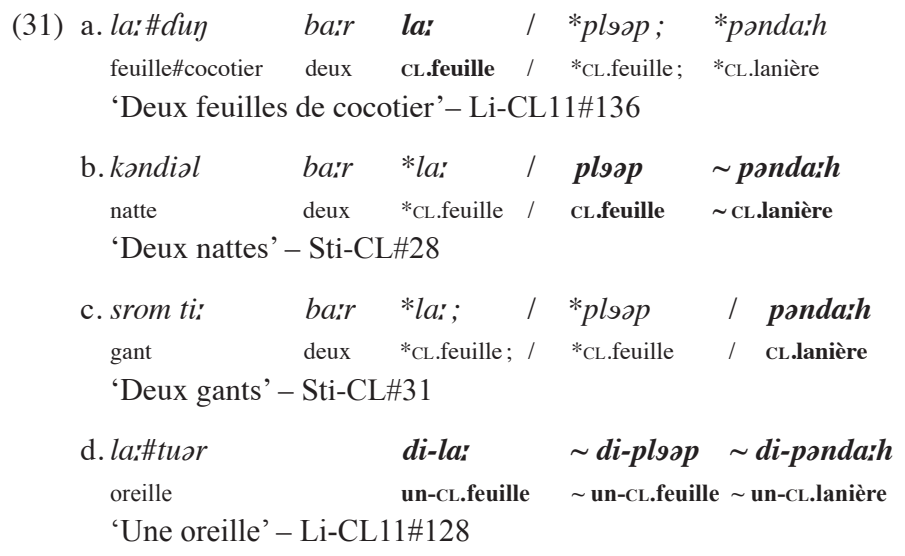

\subsubsection{Objets $3 D$ - ronds}

\section{a) Ronds : $p \varepsilon j$ - 'cL.fruit'}

On utilise le classificateur général $p \varepsilon j$ 'fruit' pour classifier les fruits, qu'ils soient gros (32a-b) ou petits (32c-d). Pour le moment, les données ne montrent aucun exemple avec des objets gros et ronds autres que des fruits.

$$
\begin{array}{ccc}
\text { (32) a. paj\#ləhoy } & \text { pej } & \text { pej } \\
\text { papaye } & \text { trois } & \text { CL.fruit } \\
\text { 'Trois papayes' } & \text { Sti-CL\#40 }
\end{array}
$$

35. Plus précisément, lanière végétale obtenue après avoir rasé le bois. 


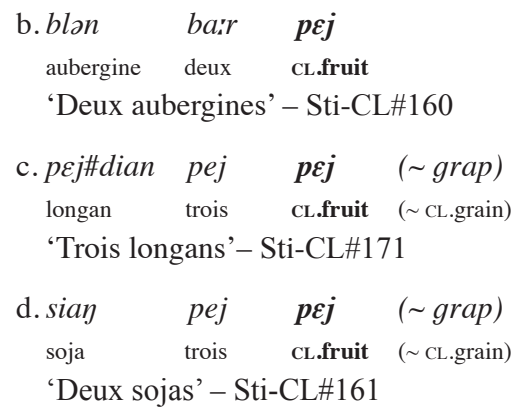

Le classificateur catégorise également des fruits non pas ronds mais longs. C'est la tridimensionnalité des objets qui semble être retenue pour la catégorisation :
a. $t o: h \quad$ pej pej $\left({ }^{*}\right.$ tэəm) haricot trois cL.fruit (*CL.tronc)
'Trois haricots' - Sti-CL\#158
b.rə.pэฤ pej pej $\left({ }^{*} t 9 ә m\right)$
concombre trois cL.fruit (*L.tronc)
'Trois concombres' - Sti-CL\#164

\section{b) Ronds et gros : mbum - 'cL.tuber'}

On utilise le classificateur spécifique mbum 'tubercule' pour classifier les tubercules (gingembre, taro, igname...). On ne peut pas utiliser le classificateur CL.fruit $p \varepsilon j$ avec ces derniers.

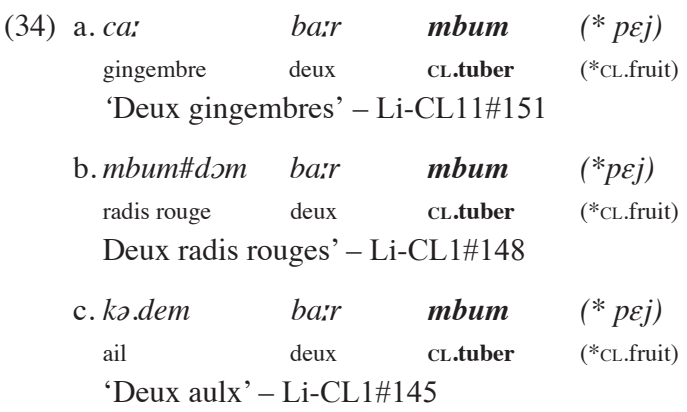




\section{c) Ronds et petits : grap - 'CL.grain'}

On utilise le classificateur grap 'grain' pour classifier les objets ronds et petits : petits fruits ${ }^{36}$ et graines (35), mais également, par extension, perles, yeux, larmes, etc. (36) :

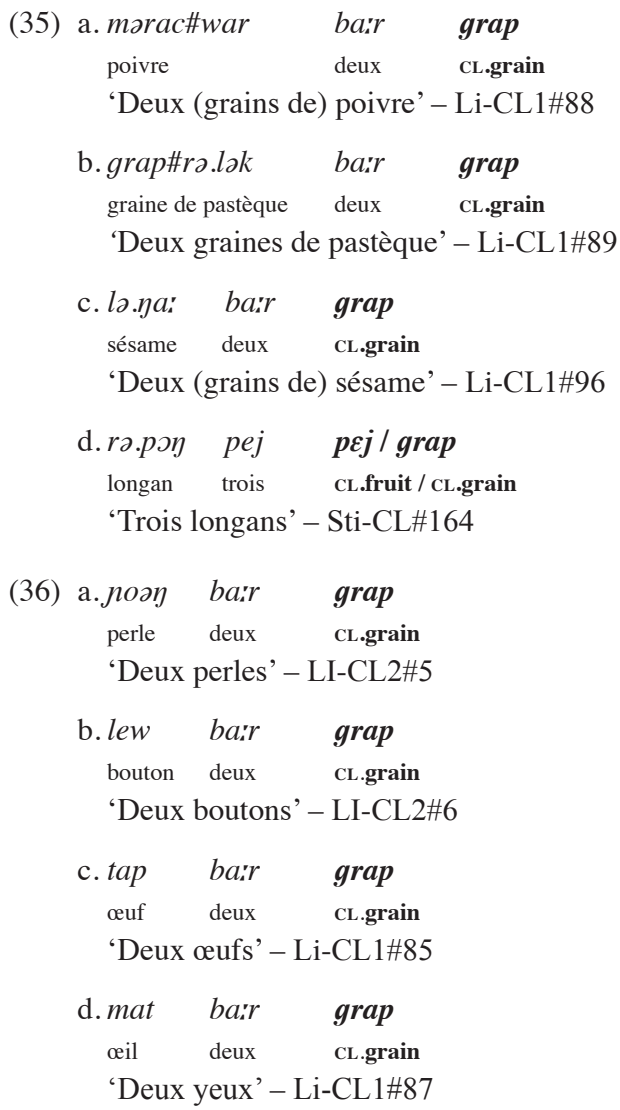

36. Qui peuvent prendre aussi bien le classificateur général $p \varepsilon j$ 'CL.fruit' que le classificateur grap 'CL.grain', cf. exemple (35d). 


\subsection{Nature des objets}

\subsubsection{Entités géographiques : kətok kəleəj - 'cl.endroit'}

Les entités géographiques (forêts, maisons, escaliers, villages, rivières, grottes, etc.) sont classifiées avec les morphèmes katวk ou kaleəan, dont l'origine lexicale signifie 'endroit'. kaleəan, un emprunt au khmer, kənlasn, est beaucoup plus fréquemment utilisé que kətək, terme natif stieng ${ }^{37}$.
a. [...] ni: bair katsk/koleagn
maison deux cL.endroit
'[...] Deux maisons' - Eli.T-Vi-JN\#4
b. gü\#haaw\#ni: di-katok/-kalean
escalier un-cL.endroit
'Un escalier' - Sti-CL\#47

\subsubsection{Machines : grian - 'cL.machine'}

Pour ce qui concerne les machines (motos, voitures, etc.), on utilise le classificateur griż, emprunté au khmer krìz utilisé avec le même emploi principal :

hej Pa:n moto ba:r griay
1sG.s avoir moto deux
'J'ai deux motos' - Eli.Kh-MP\#6

\section{Conclusion}

Cet article constitue une «nouvelle» description du système de classificateurs numéraux de tri d'une langue môn-khmère. Nous en avons souligné principalement les aspects typologiques, morphosyntaxiques et sémantiques.

Notons pour conclure que les caractéristiques de ce système sont très répandues en Asie du Sud-est, comme souligné dans la typologie de Karen Adams (1989) : avec une classe d'animés au sein de laquelle une distinction est clairement établie entre les humains et les animaux; différentes classes pour les non-animés établies sur le paramètre physique qu'est la forme (long-1D, plat-2D, rond-3D) accompagnées de critères

37. Il s'agit là d'un indice supplémentaire de l'influence du khmer dans le système des classificateurs du stieng. 
secondaires tels que la rigidité ( $v s$ flexibilité) et la taille relative; et enfin des termes relatifs aux plantes (ou à des parties de plantes) utilisés par extension métaphorique pour les classes de forme et certains paramètres secondaires (Adams, 1986b : 246-247).

Comme souligné ponctuellement dans la description sémantique, on rencontre un certain nombre de variations idiolectales. Ces variations concernent d'abord l'inclusion d'un objet dans telle ou telle classe : s'il existe un «tronc commun», un consensus entre tous les locuteurs, des désaccords se manifestent néanmoins pour certains objets moins prototypiques et situés aux frontières sémantiques des classes. On note également des divergences d'ordre pragmatique, notamment relatives au domaine des codes de politesse (cf. l'emploi de bok pour les humains, 5.1.2) ou encore d'ordre discursif (selon les besoins de la situation d'énonciation, on insiste plus sur telle caractéristique de l'objet au détriment d'une autre, ce qui implique l'emploi possible de classificateurs différents pour un même nom). Ce type de variation est commun dans les langues à classificateurs (Adams, 1986b : 244-245).

Enfin, l'influence du khmer sur ce système - influence que nous avons pu souligner à travers les quelques classificateurs empruntés ainsi que le caractère facultatif des classificateurs - caractère que nous n'avons que peu évoqué - posent plusieurs questions quant à la dynamique du système (voir Grinevald, $2002: 265$ ) :

- la position du système au sein d'une vague de diffusion plus vaste (classificateurs dans les langues d'Asie et diffusion aréale);

- son âge (récent vs ancien);

- son cycle de vie (émergent vs déclinant);

- sa productivité (actif et ouvert vs figé);

- son degré de grammaticalisation (ici en l'occurrence peu grammaticalisé).

Ces différentes problématiques d'ordre aréal et dynamique devront faire l'objet de futurs travaux sur les classificateurs du stieng. 


\section{ABRÉVIATIONS}

$\begin{array}{ll}\text { 1SG.S } & \text { '1 } 1 \text { re personne du singulier sujet' } \\ \text { 3IMPERS.S } & \text { '3e personne impersonnel sujet' } \\ \text { 3SG.POSS } & \text { '3e personne du singulier possessif' } \\ \text { ADJ } & \text { 'Adjectif' } \\ \text { ADV } & \text { 'Adverbe' } \\ \text { CL } & \text { 'Classificateur' } \\ \text { COP.LOC } & \text { 'Copule locative' } \\ \text { DEM } & \text { 'Démonstratif' } \\ \text { EXIST } & \text { 'Existentiel' } \\ \text { MS } & \text { 'Mensural' } \\ \text { N } & \text { 'Nom' } \\ \text { N.CLASSE } & \text { 'Nom de classe' } \\ \text { NUM } & \text { 'Numéral' } \\ \text { PREP } & \text { 'Préposition' } \\ \text { PRO } & \text { 'Pronom' } \\ \text { V } & \text { 'Verbe' }\end{array}$

\section{RÉFÉRENCES BIBLIOGRAPHIQUES}

Adams K. L. (1982) : Systems of Numeral Classification in the Mon-Khmer, Nicobarese and Aslian Subfamilies of Austro-asiatic, Ph.D. diss., Univ. of Michigan.

Adams K. L. (1986a): The influence of non-austroasiatic languages on numeral classification in Austroasiatic, Southeast Asia as a linguistic area Conference, Univ. of Chicago, (16 avril).

Adams K. L. (1986b) : «Numeral Classifiers in Austroasiatic», dans C. Craig (dir.), Noun Classes and Categorization: proceedings of a symposium on categorization and noun classification, Typological Studies in Language, 7, Amsterdam/Philadelphie, John Benjamins, 242-262.

AdAms K. L. (1989) : Systems of Numeral Classification in the Mon-Khmer, Nicobarese and Aslian Subfamilies of Austro-asiatic, Pacific Linguistics, series B-101, Canberra, The Australian National University.

Adams K. L. (1992) : «A comparison of the Numeral Classification of Humans in Mon-Khmer», MKS, 21, 107-129.

Adams K. L. et Conklin N. F. (1973) : «Toward a Theory of Natural Classification », Papers from the Ninth Regional Meeting, Chicago Linguistic Society, 9, 1-10.

Aikhenvald A. Y. et Green D. (1998) : «Palikur and the typology of classifiers », Anthropological Linguistics, 40, 429-480. 
Aikhenvald A. Y. (2000) : Classifiers: a typology of noun categorization devices, Oxford, Oxford University Press.

BisAng W. (1993) : «Classifiers, quantifiers and class nouns in Hmong», Studies in Language, 17/1, 1-51.

BISANG W. (1999) : «Classifiers in East and Southeast Asian languages: Counting and beyond», dans J. Gvozdanovic (dir.), Numeral types and changes worldwide, Berlin, Mouton de Gruyter, 113-185.

Croft W. (1994) : «Semantic universals in classifier systems », Word, 45, 145-171.

DeLAncey S. (1986) : «Towards a history of Thai classifiers system», dans C. Craig (dir.), Noun Classes and Categorization: proceedings of a symposium on categorization and noun classification, Typological Studies in Language, 7, Amsterdam/Philadelphie, John Benjamins, 437-452.

Dixon K. M. W. (1986) : «Noun classes and noun classification in typological perspective », dans C. Craig (dir.), Noun Classes and Categorization: proceedings of a symposium on categorization and noun classification, Typological Studies in Language, 7, Amsterdam/Philadelphie, John Benjamins, 105-112.

ERbaugh M. S. (1986) : «Taking stock: the development of Chinese noun classifiers historically and in young children», dans C. Craig (dir.), Noun Classes and Categorization: proceedings of a symposium on categorization and noun classification, Typological Studies in Language, 7 , Amsterdam/Philadelphie, John Benjamins, 399-436.

GIL D. (2011) : «Numeral Classifiers », dans M. S. Dryer et M. Haspelmath (dir.), The World Atlas of Language Structures Online, Munich, Max Planck Digital Library, feature 55A. Disponible sur <http://wals.info/ feature $/ 55 \mathrm{~A}>$.

Greenberg J. H. (1972) : «Numeral classifiers and substantival number: Problem in the genesis of a linguistic type », Working papers In Language Universals, 9, 1-39.

Goldwasser O. et Grinevald C. (2012) : «What are "Determinatives" good for?», dans E. Grossman, S. Polis et J. Winand (dir.), Lexical Semantics in Ancient Egyptian, Studia Monographica series of Lingua Aegyptia.

Grinevald C. (1999) : «Typologie des systèmes de classification nominale», Faits de langues, 14, «La catégorisation dans les langues », 101-122.

Grinevald C. (2000) : «A morphosyntactic typology of classifiers », dans G. Senft (dir.), Nominal classification, Cambridge, Cambridge University Press, 50-92.

Grinevald C. (2002) : «Making sense of nominal classification systems, noun classifiers and the grammaticalization variable », New reflections on grammaticalization, Typological Studies in Language, 49, Amsterdam/Philadelphie, John Benjamins, 259-275. 
Grinevald C. (2004) : «Classifiers», dans C. Lehmann, G. Booij et J. Mugdan (dir.), Morphology: a Handbook on inflection and Word Formation, 2, Article 97, Berlin, Walter de Gruyter, 1016-1032.

Haiman J. (2011) : Cambodian: Khmer, London Oriental and African Language Library, 16, Amsterdam, John Benjamins.

JoNES R. B. (1970) : «Classifier constructions in Southeast Asia», Journal of the American Oriental Society, 90/1, 1-12.

MiLler V. G. (1976) : An overview of Stieng grammar, SIL/UND.

Vittrant A. (2002) : «Classifier Systems and Noun Categorization Devices in Burmese», Berkeley Linguistics Society: Proceedings of the Annual Meeting, 28S, 129-148.

Thомаs D. D. (1971) : Chrau grammar, Oceanic Linguistics Special Publication, 7, Honolulu, University of Hawaii Press.

Vogel S. (2002) : «Détermination nominale, quantification et classification en khmer contemporain », BEFEO, 89, 183-201.

Vogel S. (2006) : Introduction à la langue et aux dits traditionnels des Phnong de Mondulkiri, Phnom Penh, Éditions Funan. 